\title{
Kinetics of Laser Induced Plasma on an Aqueous Surface
}

\author{
J. BEN AHMED ${ }^{a}$, Z. BEN LAKHDAR ${ }^{a}$ and G. TAIEB ${ }^{\mathrm{b}, *}$ \\ a Laboratoire de Spectroscopie Atomique-moléculaire et Applications, \\ Département de physique-Faculté des Sciences de Tunis Tunisie \\ ${ }^{b}$ Laboratoire de Photophysique Moléculaire du CNRS, Bât 210-Université Paris Sud \\ 91405, France
}

(Received 12 September 2002)

Emission spectra of $M g \mathrm{I}$ and $M g \mathrm{II}$ from a plasma induced by the interaction of a laser pulse with the surface of aqueous solutions of $\mathrm{MgCl}_{2}$, were recorded by a time resolved spectroscopy method to obtain information on the processes involved in the formation and the evolution of the two different species. A kinetic model based on ion-electron recombination produced during the relaxation of the plasma is constructed with the aim to explain the origin and the temporal shift of observed $\mathrm{Mg}^{+}$ and $\mathrm{Mg}$ emissions. Comparison with the experimental results is presented and discussed.

Key words: Plasma; Relaxation; Recombination; Kinetics; Atomic emission; Laser

\section{INTRODUCTION}

In the last decades, the Laser-Induced Plasma (LIP) technique has been widely used as an analytical tool to get information on the contents of water and organic solutions [1-3]. The technical approach consists mainly of focusing a beam of a pulsed laser on the sample to produce a transient plasma and to use atomic emission spectroscopy (AES) to analyze the solution. Two setups are used: in the first the plasma is produced within the bulk of the liquid $[4,5]$, while in the second it is produced at its surface $[6,7]$. The latter case gives more information as the plasma formed on the surface is more intense and has a longer life time. In some cases the laser beam was focused in an aerosol or in a liquid jet [8,9]. The processes in the formation of a plasma by interaction of a pulsed laser with media have been discussed in detail $[10,11]$.

To our knowledge, all studies show that, in the case of alkaline solutions, only the neutral atoms $(\mathrm{NaI}, \mathrm{KI})$ are observed by their radiative deexcita-

\footnotetext{
* Corresponding author. E-mail: guy.taieb@ppm.u-psud.fr
} 
tion, and for the alkaline-earth solutions, neutral atoms $(M g \mathrm{I}, \mathrm{CaI})$ and mono-charged ions ( $\mathrm{MgII}(\mathrm{CaII})$ are observed, whereas the spectra of $\mathrm{NaII}$, $K \mathrm{II}, \mathrm{CaIII}$ and $\mathrm{MgIII}$ are not seen. D. A. Cremers et al. [5] note that in the case of a plasma induced in a solution of $\mathrm{CaCl}_{2}$ the emission of $\mathrm{CaI}$ is observed after the emission of $\mathrm{CaII}$. The same behavior is observed in the case of $\mathrm{MgCl}_{2}$ [6]. The observations of these authors and others mentioned in our previous work [11] lead us to conclude that a electron-ion recombination process can be at the origin of the formation of the excited species. The goal of this work is to verify this hypothesis.

We have recorded the time evolution of the intensities of some $M g I I$ and $M g I$ lines emitted in a plasma induced by Nd:YAG laser pulses on the surface of water solutions of $\mathrm{MgCl}_{2}$, and we have built a kinetic model based on recombination processes. The agreement of the results given by the model with those obtained experimentally is presented and discussed.

\section{EXPERIMENTAL SET UP}

Details of the experimental set up are given elsewhere [11]: the beam of a pulsed Nd:YAG laser emitting at $532 \mathrm{~nm}$ with a pulse duration of $10 \mathrm{~ns}$ and $75 \mathrm{~mJ}$ energy is focused at a repetition rate of $2 \mathrm{~Hz}$ on the surface of an aqueous solution $\left(\mathrm{MgCl}_{2}, 4-5 \mathrm{H}_{2} \mathrm{O}, 0.05 \mathrm{Mol} / 1\right.$, Prolabo, purity $\left.97 \%\right)$. The emitted light from the central slice (the observed slice has a thickness of $100 \mu \mathrm{m}$ through the entire height of the plume) of the plasma is collected on the entrance slit of a $60 \mathrm{~cm}$ focal length monochromator, equipped with a photomultiplier (Hamamatsu R928). The temporal behavior of the emission is measured by a digital oscilloscope (Tektronix 320 TDS) and processed by a computer using a Labview program.

\section{RESULTS}

The time evolution of the plasma emission in the $2770 \AA$ to $2860 \AA$ region is shown in Figure 1. A series of lines are observed: emissions of $M g \mathrm{II}$ (2803 $\AA, 2797 \AA, 2795 \AA, 2790 \AA)$ and emissions of $M g \mathrm{I}(2777 \AA, 2852 \AA)$. Shortly after the laser pulse the dominant radiation is a strong continuum attributed to bremsstrahlung phenomena (free-free transition) and collisional radiative recombination (free-bound transition).

Figure 2 shows the time evolution of the continuum emission (at $2860 \AA$ ) together with the emission of $M g I I$ at $2803 \AA\left(3 \mathrm{~S}_{1 / 2}-3 \mathrm{P}_{1 / 2}\right)$ and of $M g \mathrm{I}$ at $2852 \AA\left(3 \mathrm{~S}_{0}-3 \mathrm{P}_{1}\right)$. The continuum emission decreases rapidly compared 


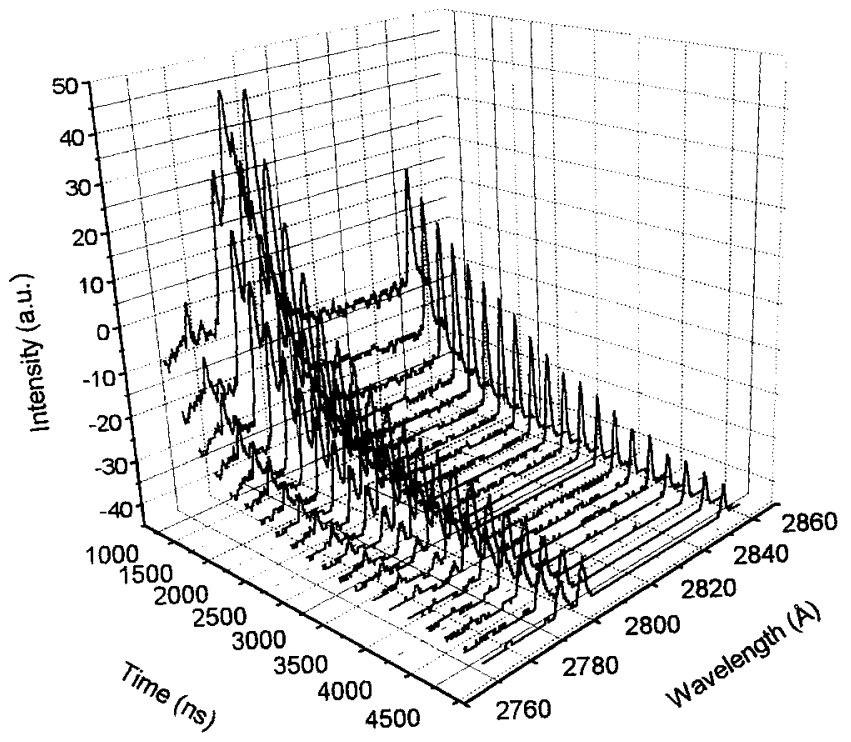

FIGURE 1 Time evolution of the spectrum of the plasma between $2770 \AA$ and $2860 \AA$.

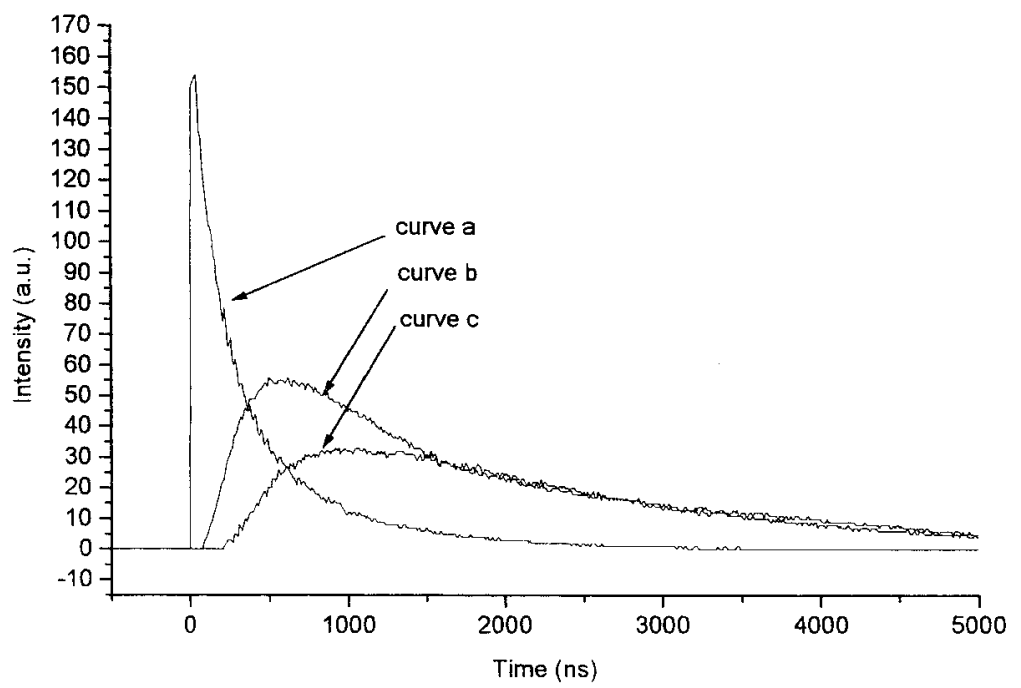

FIGURE 2 Curve a: time evolution of the background continuum at $2760 \AA$. Curve b: $M g I I$ emission at $2803 \AA$, emission at peak. Curve c: $M g \mathrm{I}$ emission at $2852 \AA$, emission at peak. Notice the delay in the appearance of the two lines. 
to the discrete emission and we can measure the onset of the ionic line which starts to be observed at $t \approx 80 \mathrm{~ns}$ while the neutral starts at $t \approx 200 \mathrm{~ns}$, with a shift of $120 \mathrm{~ns}$. In Figure 3, the scatter graph (square) shows the time evolution of the $M g I I$ line intensity at $2803 \AA$, where it should be noted that the intensity increases rapidly to reach its maximum at $t \approx 550 \mathrm{~ns}$ and then decreases: a fit with an exponential decay is shown, which is discussed below. The scatter graph (triangle) given shows the evolution of the fundamental resonance $M g \mathrm{I}$ line intensity at $2852 \AA$ : it increases to reach its maximum at $t \approx 950 \mathrm{~ns}$ before decreasing more slowly compared to the preceding one, and the fit shown will also be discussed below. In the time interval $500 \mathrm{~ns}<t<1000 \mathrm{~ns}$, we note a decrease of the $\mathrm{Mg}^{+}$emission although the emission of $\mathrm{Mg}$ continues to increase. For $t>1000 \mathrm{~ns}$, the emissions of both $\mathrm{Mg}^{+}$and $\mathrm{Mg}$ decrease at different rate.

In Figure $4 \mathrm{a}$, the temporal behavior of the lines of $M g \mathrm{I}$ at $2777 \AA$ and $2852 \AA$ are compared: apart from the relative intensities, they are quite similar, as is the temporal behavior of the lines of $M g I I$ at $2803 \AA$ and $2795 \AA$ displayed in Figure 4b. From the similarity of the time evolution of the neutral emissions, we can suppose that the excited states of $M g \mathrm{I}$ are formed by the same mechanism. Also from the similarity of the tem-

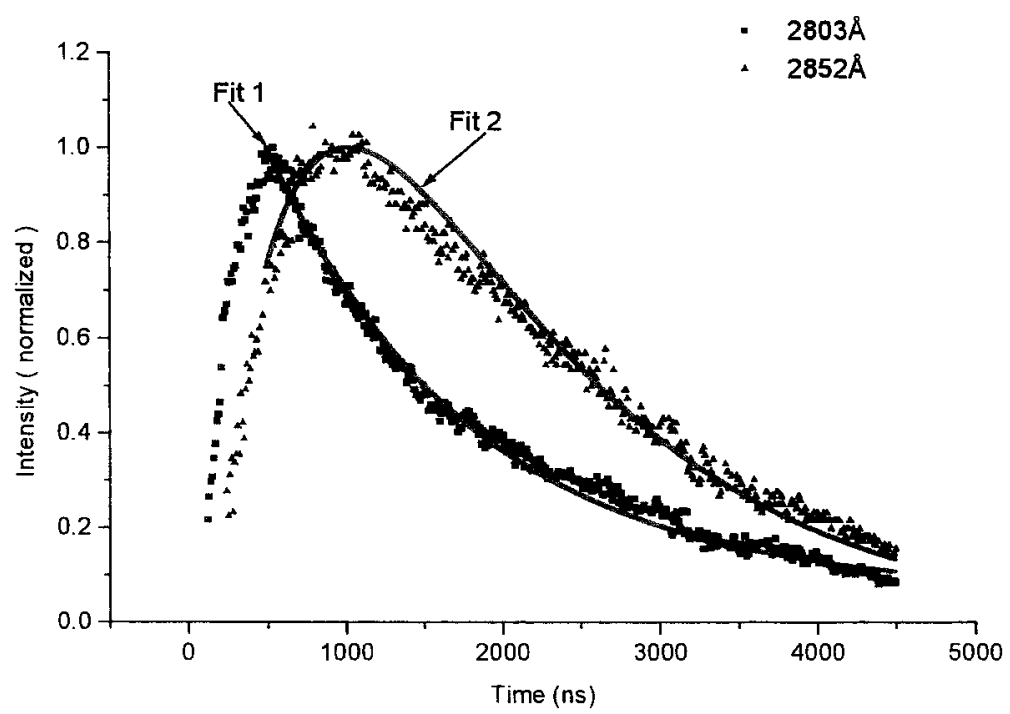

FIGURE 3 Time evolution of the $M g I I$ line intensity at $2803 \AA$ : the intensities are normalized by the intensity at $t=550 \mathrm{~ns}$. The continuous line fit 1 is an exponential decay (see text). Time evolution of the $M g I$ line intensity at $2852 \AA$ : the intensities are normalized by the intensity at $t=1100 \mathrm{~ns}$. The continuous line represents fit 2 is the sum of three exponential 


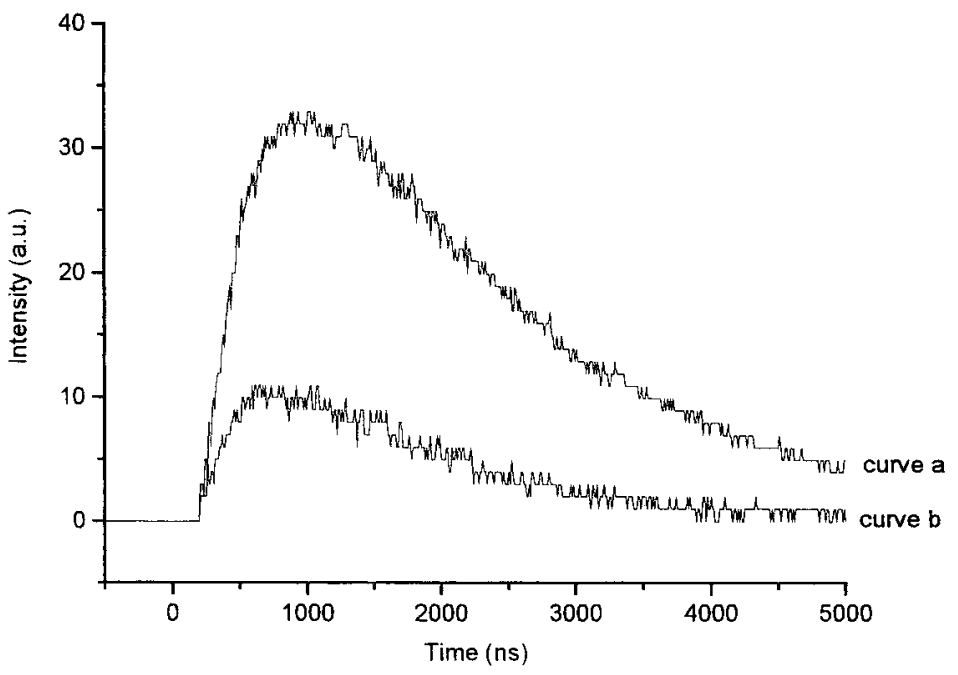

FIGURE $4 \mathrm{a}$ Temporal variation of the $M g I$ lines at $2777 \AA$ and $2852 \AA$ : the time evolution is similar for both lines.

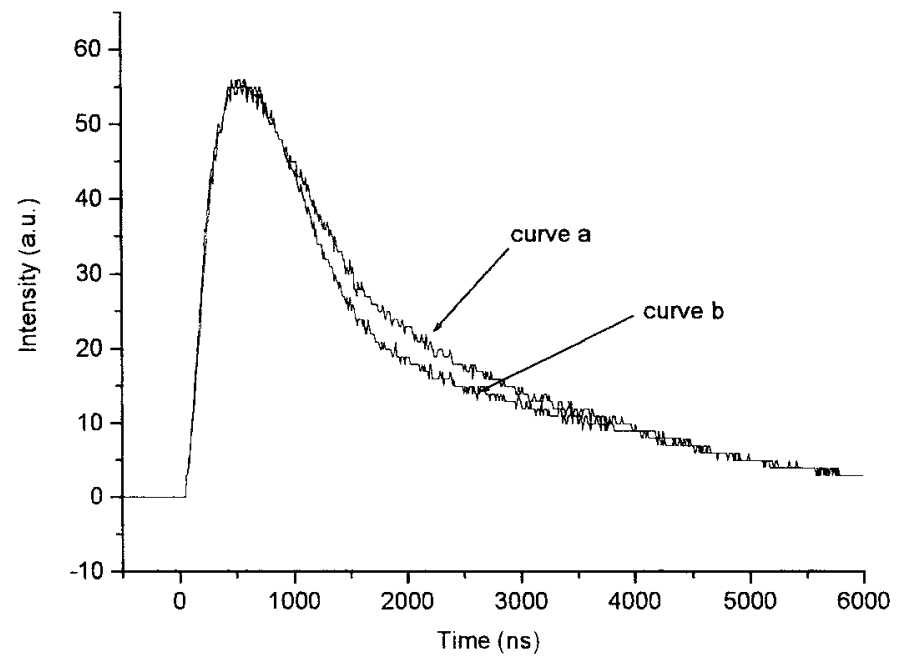

FIGURE 4b Temporal variation of the $M g I I$ lines at $2795 \AA$ and $2803 \AA$ : the time evolution is similar for both lines. 
poral behavior of the ionic emissions, we can make the same assumption for the formation of excited states of $M g I I$. Since the temporal behavior of the ionic emission is different from that of $M g I$, the process of formation of the excited states must be different.

\section{KINETIC MODEL}

It is well known that free electrons can be captured by a positive charged ion via different mechanisms [12-14].

(a) Radiative Recombination (RR): a direct capture of a free electron by an ion $X^{q+}$ where the excess energy is carried away by a photon. This phenomena contributes to the continuum spectrum emission [15-16].

$$
X^{q+}+e \rightarrow\left[X^{(q-1)+}\right]^{*}+h v
$$

(b) Three Body Re combination (TBR)

$$
X^{q+}+e+e \rightarrow\left[X^{(q-1)+}\right]^{*}+e^{*}
$$

in this case the excess energy is carried away by the second electron $e^{*}$.

(c) Dielectronic Recombination (DR): in this case the excess energy is used to excite a core electron within the ion. After this capture process the ion is in a multiply excited state which can decay in different ways [14]

$$
X^{q+}+e \rightarrow\left[X^{(q-1)+}\right]^{* *} \rightarrow\left[X^{(q-1)+}\right]^{*}+h v_{1} \rightarrow\left[X^{(q-1)+}\right]+h v_{1}+h v_{2}
$$

The processes presented (RR, TBR, DR) can be at the origin of the observed spectrally discrete line emission, and we will use that assumption in the following treatment.

In our previous work [11] we had determined the temporal evolution of the plasma generated under our experimental conditions: the electronic temperature $T_{e}$ drops from $28000 \mathrm{~K}$ at $t \approx 500 \mathrm{~ns}$ to 21000 at $t \approx 5000 \mathrm{~ns}$. The evolution of the electronic density $N_{e}$ under experimental conditions described here and elsewhere [11] can be given by the phenomenological function:

$$
N_{e}(t)=N_{0} \exp \left(\frac{t_{0}-t}{\tau_{e}}\right) \quad \text { for } t>t_{0}=500 \mathrm{~ns}
$$

where $\tau_{e}$, the time constant of relaxation of $N_{e}$, is equal to $1200 \pm 50 \mathrm{~ns}$ and $N_{0}$, the electronic density at $t=500 \mathrm{~ns}$ equal to $1.25 \times 10^{18} \mathrm{~cm}^{-3}$. 
The next step is to interpret the time evolution of the plasma after $t_{0}$, the time from which we have determined the evolution of the electronic density $N_{e}[11]$.

We can separate the time scale of the ion-electron recombination into two parallel and almost simultaneous process: process $\mathbf{1}$ is the recombination of the electrons with the $\mathrm{Mg}^{++}$ions ejected from the solution to give $\mathrm{Mg}^{+}$ ions, while process 2 is the recombination of the $\mathrm{Mg}^{+}$ions formed in the first step with electrons to give $\mathrm{Mg}$ atoms.

\section{Process 1}

$$
\mathrm{Mg}^{++}+e \stackrel{k_{1}}{\rightarrow} \mathrm{Mg}^{+*} \rightarrow \mathrm{Mg}^{+}+h v_{\mathrm{Mg}^{+}}
$$

This reaction describes the recombination processes assuming the approximation of a small variation of the electronic density. Indeed, the constant decay of the electronic density is much larger than the lifetime of exited states of $\mathrm{Mg}^{+}$.

The rate equation for the process (5) is given by:

$$
\frac{\mathrm{d}\left[\mathrm{Mg}^{+}(t)\right]}{\mathrm{d} t}=k_{1} N_{e}(t)\left[\mathrm{Mg}^{++}(t)\right]
$$

where $k_{1}$ is the recombination coefficient and $\left[\mathrm{Mg}^{++}(t)\right]$ is the concentration of $\mathrm{Mg}^{++}$ion in the plasma. It is given by:

$$
\left[\mathrm{Mg}^{++}(t)\right]=\left[\mathrm{Mg}^{++}\left(t_{0}\right)\right]-\left[\mathrm{Mg}^{+}(t)\right]
$$

Using the Saha equation, the populations $\left[\mathrm{Mg}^{+}\right],\left[\mathrm{Mg}^{++}\right]$and the electronic density $N_{e}$ are related by [17]:

$$
\frac{N_{e}\left[\mathrm{Mg}^{++}\right]}{\left[\mathrm{Mg}^{+}\right]}=\frac{2 Z_{\mathrm{Mg}^{++}}(T)}{Z_{\mathrm{Mg}^{+}}(T)}\left(\frac{m K_{B} T}{2 \pi \hbar^{2}}\right)^{3 / 2} \exp \left(-\frac{E_{\infty}^{\mathrm{Mg}^{+}}-\Delta E_{\infty}^{\mathrm{Mg}^{+}}}{K_{B} T}\right)
$$

where $Z_{\mathrm{Mg}^{++}}(T)$ and $Z_{\mathrm{Mg}^{+}}(T)$ are the partition functions, $E_{\infty}^{\mathrm{Mg}^{+}}$and $\Delta E_{\infty}^{\mathrm{Mg}^{+}}$ are respectively the ionization energy and its correction for interaction in the plasma.

The density of population $\left[\mathrm{Mg}_{u}^{+}\right]$which are in quantum states $u$ is given by the Boltzmann distribution [17]:

$$
\left[\mathrm{Mg}_{u}^{+}\right]=\left[\mathrm{Mg}^{+}\right] \frac{g_{u}}{Z(T)} \exp \left(\frac{E_{u}}{K_{B} T}\right)
$$


The emission coefficient $I_{u l}$ of a line corresponding to the transition between levels $u$ and $l$ is given by:

$$
I_{u l}=\frac{h v_{u l}}{4 \pi} \mathrm{A}_{u l}\left[\mathrm{Mg}_{u}^{+}\right] P(v) .
$$

In the work presented elsewhere [11], we have shown that the temperature $T$ does not vary significantly in the range $500 \mathrm{~ns}<t<5000 \mathrm{~ns}$. Therefore, we can assume with a good approximation that the second term in Eq. (8) is constant.

By combining Eqs. (8), (9) and (10) we can write:

$$
I_{u l}, \alpha N_{e}\left[\mathrm{Mg}^{++}\right]
$$

By observing the temporal evolution of the line intensity at $2803 \AA$ of $\mathrm{Mg}^{+}$ given in Figure 3, we note that the intensity decreases exponentially for $t>500 \mathrm{~ns}$ with a decay constant $\tau \approx 1285 \mathrm{~ns}$. This was also observed for $\mathrm{Ca}^{+}$lines [11]. We notice that this constant decay is similar to the constant decay of the electronic density. This implies that in the expression $10 \mathrm{~b}$ $\left[\mathrm{Mg}^{++}\right]$remains constant during the observation time.

In the following treatment, we assume that $\left[\mathrm{Mg}^{++}(t)\right] \approx\left[\mathrm{Mg}^{++}\left(t_{0}\right)\right]$

$$
\text { (6) } \begin{aligned}
& \Rightarrow\left[\mathrm{Mg}^{+}(t)\right]=\int_{t_{0}}^{t} k_{1}\left[\mathrm{Mg}^{++}\left(t_{0}\right)\right] N_{0} \exp \left(\frac{t_{0}-t^{\prime}}{t_{e}}\right) \mathrm{d} t^{\prime} \\
& \Rightarrow \mathrm{Mg}^{+}(t)=k_{1}\left[\mathrm{Mg}^{++}\left(t_{0}\right)\right] N_{0} \tau_{e}\left(1-\left(\frac{t_{0}-t}{t_{e}}\right)\right)
\end{aligned}
$$

$\left[\mathrm{Mg}^{+}(t)\right]$ represents the concentration of $\mathrm{Mg}^{+}$ion formed by recombination between $t_{0}$, and $t$.

$\left[\mathrm{Mg}^{+*}(t)\right]$ is given by

$$
\left[\mathrm{Mg}^{+*}(t)\right]=\int_{t-\tau_{a}}^{t} k_{1}\left[\mathrm{Mg}^{++}\left(t_{0}\right)\right] N_{0} \exp \left(\frac{t_{0}-t^{\prime}}{\tau_{e}}\right) \mathrm{d} t^{\prime}
$$

where $\tau_{a}$ is the lifetime of the excited species: it is equal respectively to $3.8 \mathrm{~ns}$ and $2 \mathrm{~ns}$ for upper states corresponding to the emissions $2803 \AA$ of $\mathrm{Mg}^{+}$and $2852 \AA$ of $\mathrm{Mg}$.

$$
(12) \Rightarrow\left[\mathrm{Mg}^{+*}(t)\right]=k_{1}\left[\mathrm{Mg}^{++}\right] N_{0} \tau_{e} \exp \left(\frac{t_{0}-t}{\tau_{e}}\right)\left\{\exp \left(\frac{\tau_{a}}{\tau_{e}}\right)-1\right\}
$$


Using $\tau_{a} \ll \tau_{e}$ :

$$
\left[\mathrm{Mg}^{+*}(t)\right]=k_{1}\left[\mathrm{Mg}^{++}\right] N_{0} \tau_{a} \exp \left(\frac{t_{0}-t}{\tau_{e}}\right)
$$

The deexcitation of $\mathrm{Mg}^{+*}$ can be achieved by a radiative transition from one of its excited $p$ states to a level $l$. The line intensity $I_{p l}(t)$ is given by:

$$
I_{p l}(t)=\frac{h v_{p l}}{4 \pi} \mathrm{A}_{p l}\left[\mathrm{Mg}^{+*}(t)\right]
$$

where $A_{p l}$ is the transition probability which gives

$$
I_{p l}(t)=I_{0} \exp \left(\frac{t_{0}-t}{\tau_{e}}\right)
$$

\section{Process 2}

$$
\mathrm{Mg}^{+}+e \stackrel{k_{2}}{\rightarrow} \mathrm{Mg}^{*} \rightarrow \mathrm{Mg}+h v_{M g}
$$

This reaction describes the recombination processes assuming the approximation of a small variation of the electronic density, as for process 1.

The rate equation for the process (17) is given by:

$$
\frac{\mathrm{d}[\mathrm{Mg}(t)]}{\mathrm{d} t}=k_{2} N_{e}(t)\left[\mathrm{Mg}^{+}(t)\right]
$$

where $\left[\mathrm{Mg}^{+}(t)\right]$ represents the concentration of $\mathrm{Mg}^{+}$at the instant $t$. We assume that the concentration of $\mathrm{Mg}^{+}$at the instant $t_{0}$ is $C_{0}$.

Using (11b): $\mathrm{Mg}^{+}$is given by:

$$
\begin{aligned}
& {\left[\mathrm{Mg}^{+}(t)\right]=C_{0}+k_{1}\left[\mathrm{Mg}^{++}\left(t_{0}\right)\right] N_{0} \tau_{e}\left(1-\exp \left(\frac{t_{0}-t}{\tau_{e}}\right)\right)} \\
& \frac{\mathrm{d}[\mathrm{Mg}(t)]}{\mathrm{d} t}=k_{2} N_{e}(t)\left(C_{0}+k_{1}\left[\mathrm{Mg}^{++}\right] N_{0} \tau_{e}\left(1-\exp \left(\frac{t_{0}-t}{\tau_{e}}\right)\right)\right)
\end{aligned}
$$


and $\left[\operatorname{Mg}^{*}(t)\right]$ is given by:

$$
\begin{aligned}
{\left[\mathrm{Mg}^{*}(t)\right] } & =\int_{t-\tau_{a}}^{t} k_{2} N_{e}\left(t^{\prime}\right)\left(C_{0}+k_{1}\left[\mathrm{Mg}^{++}\right] N_{0} \tau_{e}\left(1-\exp \left(\frac{t_{0}-t^{\prime}}{\tau_{e}}\right)\right)\right) \mathrm{d} t^{\prime} \\
{\left[\mathrm{Mg}^{*}(t)\right] } & =k_{2} N_{0} C_{0} \tau_{a} \exp \left(\frac{t_{0}-t}{\tau_{e}}\right)+k_{1} k_{2}\left[\mathrm{Mg}^{++}\right] N_{0}^{2} \tau_{a} \tau_{e} \\
& \times\left(\exp \left(\frac{t_{0}-t}{\tau_{e}}\right)-\exp \left(\frac{2\left(t_{0}-t\right)}{\tau_{e}}\right)\right)
\end{aligned}
$$

We introduce $I_{m n}(t)$ as the line intensity emitted by $\mathrm{Mg}^{*}$ by a radiative transition from a level $m$ to a level $n$, given by:

$$
I_{m n}(t) \frac{h v_{m n}}{4 \pi} \mathrm{A}_{m n}\left[\mathrm{Mg}^{*}(t)\right]
$$

which gives

$$
I_{m n}(t)=I_{0} \exp \left(\frac{t_{0}-t}{\tau_{e}}\right)+I_{1}\left(\exp \left(\frac{t_{0}-t}{\tau_{e}}\right)-\exp \left(\frac{2\left(t_{0}-t\right)}{\tau_{e}}\right)\right)
$$

The expression (24) can be obtained in another way, by applying the Saha Eq. (8) to the population of $\mathrm{Mg}, \mathrm{Mg}^{+}$and $N_{e}$, by applying the Boltzmann Eq. (9) to $\mathrm{Mg}$, and by using the Eqs. (10) and (19).

\section{DISCUSSION}

In the case of a system where the electronic density follows the expression (4) and whose evolution can be described with the approximations given in Section 4, the intensity of the line emitted by an element formed after one recombination $\left(\mathrm{Mg}^{+}\right)$is given by (16) and the intensity of the line emitted by an element formed after two successive recombinations $(\mathrm{Mg})$ is given by (24).

In the case of $\mathrm{Mg}^{+}$emission at $2803 \AA$, we have used the expression (16) to fit the observed line intensity. We have adjusted $\tau_{e}$ within the experimental error, in order to obtain the best fit for $t>t_{0}$, and found $\tau_{e}=1285 \mathrm{~ns}$ (Fig. 3 fit 1 ). This value is similar to that found for the emission line of $\mathrm{Ca}^{+11}$. The striking observation is the similarity between the decay constant of the electronic and the decay constants of $\mathrm{Mg}^{+}$(and $\mathrm{Ca}^{+}$) emissions. This result allows one to conclude that the recombination process can be at the origin of $\mathrm{Mg}^{+}$(and $\mathrm{Ca}^{+*}$ ) formation. 
For the $\mathrm{Mg}$ emission at $2852 \AA$, we have used the expression (24) to fit the observed line intensity. As this intensity shows a maximum for $t>500 \mathrm{~ns}$, we have adjusted $\tau_{e}$ in order to fit this maximum with that found experimentally, we found $\tau_{e}=1300 \mathrm{~ns}$ similar to that found above (Fig. $3 \mathrm{fit}$ $2)$. By comparing the decay of the line intensities with the decay of the electronic density we obtain $\delta \tau_{e} / \tau_{e}=0.04$ where $\delta \tau_{e}$ is the difference between the value obtained from the measurement from $N_{e}$ with the one obtained with this fit; this $4 \%$ discrepancy can be considered as negligible.

The temporal behavior of $N_{e}$ and $\mathrm{Mg}, \mathrm{Mg}^{+}$is expected to be due to both plasma expansion and recombination processes. By comparing the results given by the kinetics model which is built disregarding the expansion, with those found experimentally, we note a very good agreement: a strong correlation between the time evolution of $\mathrm{Mg}^{+}$emissions and $N_{e}$ is observed and the time evolution of the $\mathrm{Mg}$ emission can be fitted by the expression obtained by the model which implies the recombination process $\mathrm{Mg}^{+}$electron. These observations lead to the conclusion that either the plasma expansion does not modify significantly the concentration of the three species within the observed zone and during the range time $(500 \mathrm{~ns}<t<5000 \mathrm{~ns})$, or that this expansion affects in a similar way the three populations.

\section{CONCLUSION}

In this work a kinetics model based on electron-ion recombination is presented and compared with the experimental results obtained from a plasma induced by a pulsed laser on the surface of water solutions of $\mathrm{MgCl}_{2}$. The time evolution of the lines intensities (2803 $\AA$ ) of $M g I I$ and (2852 $\AA$ ) of $M g I$ is reported. It is noted that the temporal behavior is different: the time of appearance, the time when they reach a maximum and the way of relaxation. By comparing with the model presented we can say that the recombination of the electrons created at the beginning of the interaction with the laser pulse, with ions ejected from the solution could be the origin of the observed exited species. In a first approximation, the kinetics of recombination electron-ion processes can be simulated with the present simple model in the observed region of the plasma and in the indicted time interval.

A.J. Mayne is acknowledged for the reading of the manuscript. Support from the cooperation program CMCU between the University of Tunis II and the University of Paris XI is acknowledged. 


\section{References}

[1] Ciucci, A., Corsi, M., Palleschi, V., Rastelli, S., Salvetti, A. and Tagoni, E. (1999) Appl. Spectrosc., 53, 960.

[2] Docchio, F., Regondi, P., Malcolm, R. C. and Mellerio, J. (1988) Appl. Opti., 27, 3661.

[3] Sun, Q., Tran, M., Smith, B. W. and Winefordner, J. D. (1999) Canadian Journal of Analytical Sciences and Spectroscopy, 44, 164.

[4] Escarugel, A., Ferhat, B., Lesage, A. and Richou, J. (2000) Journal of Quantitative Spectroscopy Radiation Transfer, 64, 353.

[5] Cremers, D. A., Radziemski, L. J. and Loree, T. R. (1984) Appl. Spectrosc., 38, 721.

[6] Angelo, C. D., Gomba, J., Iriarte, D. and Bertuccelli, G. (1999) SPIE, 3572, 534.

[7] Wachter, J. R. and Cremers, D. A. (1987) Appl. Spectrosc., 41, 1042.

[8] Ng, C. W., Ho, F. and Cheung, H. (1997) Appl. Spectrosc., 51, 976.

[9] Biswas, A., Latifi, H., Radziemski, L. J. and Armstrong, R. L. (1988) Appl. Opt., 27, 2386.

[10] Radziemski, L. J., Loree, T. R., Cremers, D. A. and Hoffman, N. M. (1983) Anal. Chem., $\mathbf{5 5}, 1246$.

[11] Ahmed, J. B., Terzi, N., Lakdhar, Z. and Taieb, G. Temporal characterization of a plasma produced by interaction of laser pulses with water solutions. Precedent paper.

[12] Bates, D. R. and Dalgarno, A. (1962) Atomic and Molecular Processes. New York: Academic Press, p. 259.

[13] Zhang, H. L. (2001) Phy. Rev. A, 64, 32719.

[14] Jacobs, V. L., Behar, E. and Rozsnyai, B. F. (2001) Journal of Quantitative Spectroscopy \& Radiation Transfer, 71, 397.

[15] Hffknecht, A., Uwira, O., Schennach, S., Frank, A. and Haselbauer, J. (1998) J. Phy. B, 31, 2415.

[16] Marr, G. V. (1964) Plasma Spectroscopy. Amsterdam, London, New York: Elsevier Publishing Company, p. 283-288.

[17] Griem, H. R. (1964) Plasma Spectroscopy. New York: McGraw-Hill, pp. 134-137 and 170. 